\title{
Proposed Enhancement of Side-Mode Suppression Ratio in $\lambda / 4$ Shifted Distributed Feedback Lasers with Nonuniform Diffused Quantum Wells
}

\author{
S. F. Yu and E. Herbert Li, Senior Member, IEEE
}

\begin{abstract}
An enhancement of the side mode suppression ratio, by utilizing interdiffused quantum wells, of a $\lambda / 4$ shifted distributed feedback laser is demonstrated theoretically. It is found that by introducing a diffusion step along the longitudinal direction of the quantum-well active region, the suppression ratio can be improved significantly for large $\kappa L(>2.6)$ devices. The maximum power for single longitudinal mode operation is increased by more than $50 \mathrm{~mW}$.
\end{abstract}

\section{INTRODUCTION}

$\mathbf{H}^{1}$ IGH-POWER $\lambda / 4$ shifted AlGaAs-GaAs distributed feedback (DFB) lasers with stable single longitudinal mode operation are well suited for wavelength-selective applications such as frequency doubling and atomic spectroscopy. However, multimode operation is frequently observed, in particular for devices with large $\kappa L(>1.25)$; this is caused by an nonuniform distribution of the refractive index and which is a consequence of the longitudinal carrier spatial hole burning (here after known as hole burning) [1]. Although $\kappa L$ $=1.25$ was found to be an optimum value for the $\lambda / 4$ shifted DFB lasers in order to maintain high stability in the single longitudinal mode operation [1], higher $\kappa L$ devices is far more attractive because of the lower threshold current density, relatively larger AM bandwidth, smaller linewidth-power products as well as lower reflection sensitivity [2]. Therefore it is highly desired to maintain high power operation with a stable single longitudinal mode in the large $\kappa L$ devices. In this letter, a simple thermally diffused quantum-well (DFQW) structure is proposed to enhance the stable longitudinal mode operation of $\lambda / 4$ shifted DFB lasers with large $\kappa L$. The active region of this laser structure consists of an nonuniform (step change in the extent of interdiffusion) DFQW structure along the longitudinal direction of the device. This will create an nonuniform stepped refractive index profile in the longitudinal direction and its purpose is to compensate the hole burning effect.

The multimode operation in the $\lambda / 4$ shifted DFB lasers is caused by excitation of the band-edge modes and which can be explained as follows [2]. As hole burning takes place in large $\kappa L(>1.25)$ devices, a concave-up longitudinal refractive index profile results which improves the longitudinal confinement of the intensity profile and reduces the gain requirement; these

Manuscript received October 16, 1995.

The authors are with the Department of Electrical and Electronic Engineering, University of Hong Kong, Pokfulam Road, Hong Kong.

Publisher Item Identifier S 1041-1135(96)02611-0. enhance the shorter wavelength band-edge mode. In a similar fashion, it is also true for the small $\kappa L(<1.25)$ devices where the longer wavelength band-edge mode enhances. From the above phenomena, the stable single longitudinal mode operation can be achieved in $\lambda / 4$ devices provided that the longitudinal refractive index profile of the active region can be alternated. In fact, it is possible to vary the refractive index profile of the active region by introducing QW materials of different band-gap's, which can be achieved by modifying the interdiffusion length of the DFQW's [3].

The device performance is analyzed here by modeling the material optical gain and refractive index of the DFQW's under external carrier injections. The material considered in our analysis is the as-grown and interdiffusion modified $\mathrm{Al}_{0.3} \mathrm{Ga}_{0.7} \mathrm{As}-\mathrm{GaAs}$ single $\mathrm{QW}$ structure. The TE polarized optical gain, $g(\lambda)$, at room temperature is calculated by the density matrix approach. The DFQW can be modeled by an error function and the extent of interdiffusion is characterized by a diffusion length, $L_{d}$ (where $L_{d}=0$ represents the asgrown $\mathrm{QW}$ ). The details of these calculations can be found in [4] and [5]. At a particular $L_{d}$ and at an external carrier injection level, $N$, the TE net optical gain spectrum, $G$, is found to have a simple expression,

$$
G(\lambda)=a(\lambda) \ln \left(N / N_{0}\right)
$$

where $\mathrm{a}(\lambda)$ is a fitted parameter, $N_{0}$ is the carrier concentration at transparency, and $\lambda$ is the photon wavelength.

The refractive index, $n_{r}(\lambda)$, of the DFQW without external injection can be calculated by using the method given in [3]. Furthermore the carrier induces refractive index change, $\Delta n_{r}$, which varies with the background refractive index profile of the active region, can be obtained from $\Delta g$ through the Kramers-Krönig dispersion relation [6], and it can be shown that at a particular $L_{d}$ the relation between $\Delta n_{r}$ and $N$ can be expressed as

$$
\Delta n_{r}(\lambda)=\left(N-N_{0}\right) d_{1}(\lambda),
$$

where $d_{1}$ is a fitted parameter.

The $\lambda / 4$ shifted DFB laser structure under investigation is shown in Fig. 1. The laser is composed of six layers; $\mathbf{n}^{+}-$ GaAs substrate, n-GaAs buffer layer, n-AlGaAs guiding layer in which the grating is defined, followed by an active QW's layer, and finally the $\mathrm{p}-\mathrm{AlGaAs}(\cong 0.2 \mu \mathrm{m})$ and $\mathrm{p}^{+}$-GaAs $(\leq 0.3 \mu \mathrm{m})$ layers which form the cladder and contact. The 


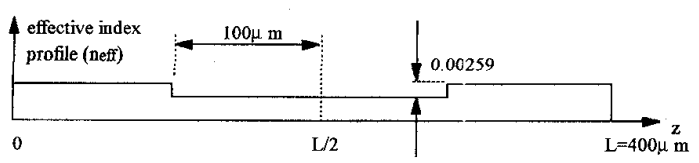

(a)

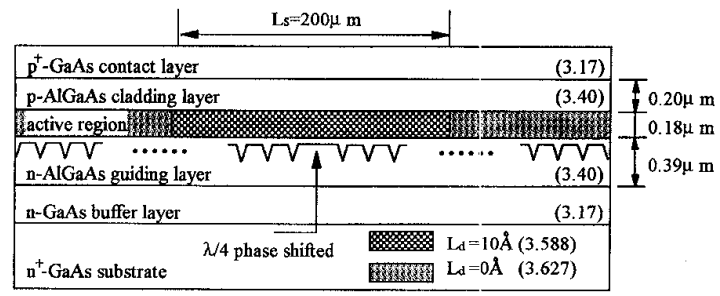

(b)

Fig. 1. (a) The built-in refractive index profile of the QW active region due to a step change of interdiffusion length along the longitudinal direction. (b) Schematic diagram of a $\lambda / 4$ DFB laser. It is assumed that the $\lambda / 4$ phase shift is located at the center of the device. $L_{s}$ is the longitudinal length for the step change ( $L_{d}=10 \AA$ ) region.

active layer is regrown on top of the guiding layer which consists of three $\mathrm{Al}_{0.3} \mathrm{Ga}_{0.7} \mathrm{As}-\mathrm{GaAs}$ single DFQW's with well and barrier thickness of $100 \AA$ and $250 \AA$, respectively. It is assumed that the active region has $L_{d}=0$ except near the $\lambda / 4$ phase shifted region where a step change of $L_{d}(\cong 10$ $\AA$ ) is introduced for a longitudinal length of $L_{s}=200 \mu \mathrm{m}$, and where the total longitudinal length of the laser cavity, $L$, is equal to $400 \mu \mathrm{m}$.

The operation wavelength of the laser is chosen to be $\lambda_{o p}=0.843 \mu \mathrm{m}$, which is the effective peak gain wavelength of DFQW in the active region. The refractive index of the DFQW, $n_{r}\left(\lambda_{o p}\right)$ for the case of $L_{d}=0$ and $10 \AA$ are equal to 3.627 and 3.588 , respectively. The values of $\mathrm{a}\left(\lambda_{o p}\right)$ and $N_{o}$ for the case of $L_{d}=0$ are equal to $689 \mathrm{~cm}^{-1}$ and $2.485 \times$ $10^{18} \mathrm{~cm}^{-3}$, and for the case of $L_{d}=10 \AA$ they are equal to $520.58 \mathrm{~cm}^{-1}$ and $2.125 \times 10^{18} \mathrm{~cm}^{-3}$, respectively. The parameter $d_{1}\left(\lambda_{o p}\right)$ given in (2) is equal to $-0.004 \times 10^{-18}$ $\mathrm{cm}^{3}$ for $L_{d}=0 \AA$ and $-0.0035 \times 10^{-18} \mathrm{~cm}^{3}$ for $L_{d}=10 \AA$.

Interdiffusion of QW's requires the penetration of impurities or vacancies through the contact and cladding layers into the active region. The contact and cladding layers form a blocking layer of the diffusion process. However, it has been shown that even the thickness of the blocking layer has dimension of $1 \mu \mathrm{m}$ [7], interdiffusion of QW's can be easily achieved. Furthermore, the diffusion of impurities also modifies the refractive indices of the contact and cladding layer and its change is in the order of roughly 0.01 . This value is relatively small and which has negligible effect on the optical confinement in the transverse direction of the waveguide.

The propagation of forward and reverse fields, $F$ and $R$, along the laser cavity are described by their time-dependent coupled optical-wave-equations as follows:

$$
\begin{aligned}
\left(\frac{1}{\nu_{g}} \frac{\partial}{\partial t} \pm \frac{\partial}{\partial z}\right)\left[\begin{array}{l}
F \\
R
\end{array}\right]= & \left(\Gamma G / 2+i \delta \beta-\alpha_{s}\right)\left[\begin{array}{l}
F \\
R
\end{array}\right] \\
& +i \kappa\left[\begin{array}{l}
F \\
R
\end{array}\right]+\left[\begin{array}{l}
j_{f s} \\
j_{r s}
\end{array}\right],
\end{aligned}
$$

where $\kappa$ is the coupling coefficient, $\alpha_{s}\left(=20 \mathrm{~cm}^{-1}\right)$ is the total absorption and scattering loss of the QW waveguide, $\Gamma(=0.35)$ is the optical confinement factor in the transverse direction, $j_{r s, f s}$ is the spontaneous emission, and $\nu_{g}(=c / 3.6)$ is the group velocity where $c$ is the velocity of light in vacuum. The deviation from Bragg's condition, $\delta \beta$, is given as

$$
\delta \beta=\frac{2 \pi}{\lambda_{o p}}\left(n_{e f f}+\Delta n_{r} \Gamma\right)-\frac{\pi}{\Lambda},
$$

where $\Lambda(=0.127 \mu \mathrm{m})$ is the period of grating and $n_{\mathrm{eff}}$ is the effective refractive index of the grated waveguide. $n_{\mathrm{eff}} \cdot$ is found to be equal to 3.302938 and 3.300346 for the DFQW's with $L_{d}=0$ and $10 \AA$, respectively. The time dependent carrier concentration profile, $N(z, t)$, along the longitudinal direction, $z$, of the active region is described by

$$
\frac{d N(z, t)}{d t}=\frac{J(t)}{q d}-\frac{N(z, t)}{\tau}-\nu_{g} G P(z, t),
$$

where $J$ is the current density, $\tau(=3 \mathrm{~ns})$ is the carrier lifetime, $d(=0.18 \mu \mathrm{m})$ is the thickness of the DFQW's active region, $q$ is the electron charge, and $P$ is the photon density. The static and dynamic behavior of the laser device can be obtained by solving the coupled wave equations (3) and the rate equation (5) of carrier in a self-consistent manner.

Fig. 2(a) shows the variation of the side-mode suppression ratio (SMSR) with normalized (by the threshold) current density, $J / J_{t h}$, for the $\lambda / 4$ shifted devices with $\kappa L=2.8$ and 3.2. It is assumed that the facets are anti-reflection coated and current is uniformly injected along the laser cavity. The corresponding maximum output power for the uniform $\left(L_{d}=\right.$ $0 \AA$ ) devices in single longitudinal mode operation (defined by a drop of SMSR from 40 to $10=30 \mathrm{~dB}$ ) are equal to 14 $\mathrm{mW}(\kappa L=2.8)$ and $5 \mathrm{~mW}(\kappa L=3.2)$. However, it is clearly seen that the introduction of the built-in refractive index step profile increases the SMSR, as these devices maintain single longitudinal mode operation up to at least $50 \mathrm{~mW}$. Fig. 2(b) shows the refractive index profile for both the uniform and step-diffused devices with $\kappa L=3.2$ at high power. The effects of hole burning on the refractive index profile are effectively minimized by the step change of $L_{d}$, and the coupling strength between the longitudinal optical intensity profile of the short wavelength band-edge mode and the hole burning of carriers is also reduced. The influence of longitudinal heat distribution on the refractive index profile is also analyzed by the model given in [8]. It is shown that the variation of temperature is less than $2 \mathrm{~K}$ and therefore can be ignored in our calculation.

In terms of the dynamic response, in particular for $\lambda / 4 \mathrm{DFB}$ lasers under large signal modulation, it has been shown that hole burning is not taken place during the first overshoot of the output power and single longitudinal mode operation is maintained. However, the built-in refractive index step may reduces the gain requirement of the band-edge modes as well as a single longitudinal mode operation. Therefore, it is necessary to consider the influence of built-in step reflective index profile on the spectral purity of the turn-on transient signal. Fig. 3(a) shows the output power spectra at the first overshoot of the step-diffused device $\left(\kappa L=2.8, L_{d}=0 \AA\right.$ । $10 \AA$ ) for two modulated currents $J=2.0 J_{t h}$ and $4.5 J_{t h}$. The 


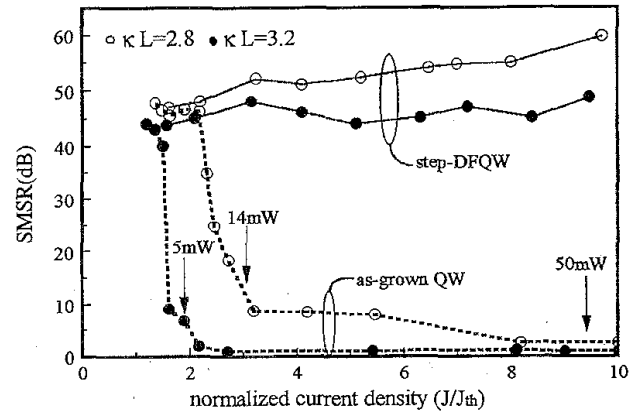

(a)

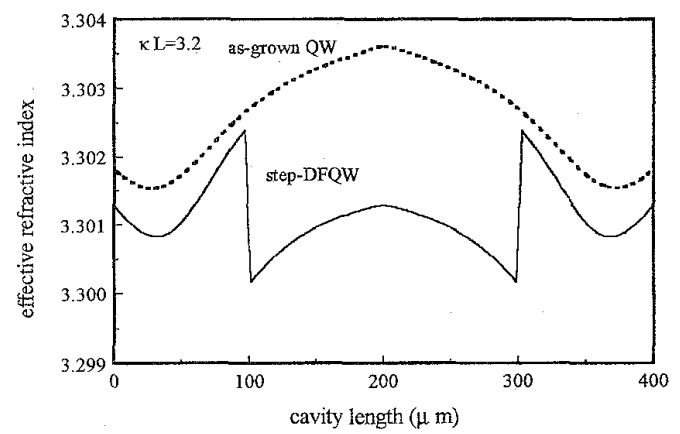

(b)

Fig. 2. (a) The side mode suppression ratio (SMSR) varies with the normalized external injection current, $J / J_{t h}$, for lasers with $\kappa L=2.8(\mathrm{O})$ and 3.2 ( ). The solid and dotted lines represent cases for the step-diffused devices $\left(L_{d}=0 \AA \mid 10 \AA\right)$ and the uniform $\left(L_{d}=0 \AA\right)$ devices, respectively. (b) The longitudinal refractive index profile of the lasers with notation as in (a). The devices are biased at 8 times of its threshold value.

$\kappa L=2.8$ device is biased initially at the threshold and then modulated by a step current. It is observed that a side mode is excited during the turn-on transient interval for the larger $J$ 's and which should impose a limitation to the modulation depth requirement. SMSR of the first overshoot power spectra are also shown in Fig. 3(b) as a function of the normalized current density for the step-diffused devices with $\kappa L=2.8$ and 3.2. The results show that no side mode is excited for the case of $\kappa L=3.2$ and, for the $\kappa L=2.8$ case, domination of side mode prevails (SMSR $<30 \mathrm{~dB}$ ) at larger current densities.

In conclusion, enhancement of SMSR is predicated theoretically by using a step change of interdiffusion length along the longitudinal direction in $\lambda / 4$ shifted DFB lasers with DFQW active region. Results show that large $\kappa L$ devices with a step refractive index profile exhibit stable as well as singlelongitudinal-mode operation for the static situation. This can be achieved by the introduction of impurities diffuse into the active region (consists of QW's) of a $\lambda / 4$ DFB laser. A practical device structure (with typical dimensions) has also been proposed and which can be realized by existence technologies. In addition, the diffusion of impurities is estimated to has a negligible effect on the guiding condition of the optical waveguide. However care must be taken to ensure a single mode operation under signal modulation situations by applying a modulation current not more than twice the $J_{t h}$ and a reasonably large enough $\kappa L$. The other advantages for

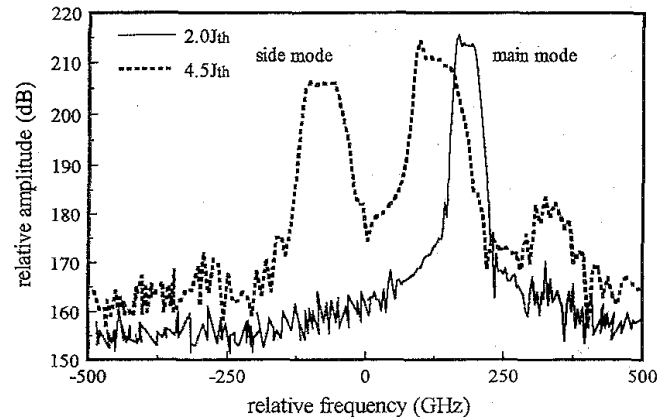

(a)

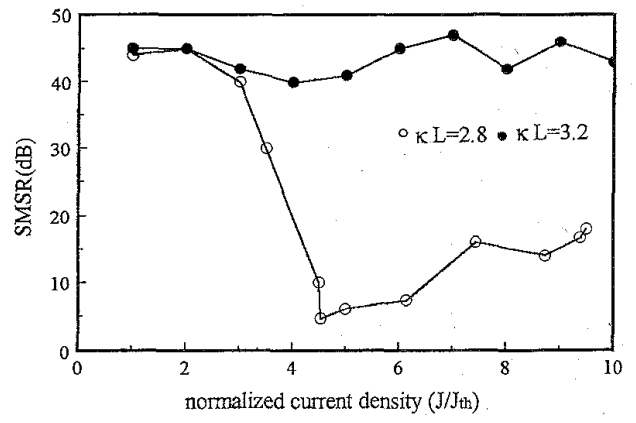

(b)

Fig. 3. (a) Output power spectra at the first overshoot of the step-diffused device ( $\kappa L=2.8, L_{d}=0 \AA 10 \AA$ ). The device is modulated by a step current with magnitude, $J=2.0 J_{t h}$ and $4.5 J_{t h}$. (b) SMSR of the first overshoot power spectrum varies with the normalized magnitude of the step current, $y / J_{t h}$, for the step-diffused device with $\kappa L=2.8(\bigcirc)$ and $3.2(\bullet)$

adopting DFQW structure are: 1) tunability of the operating wavelength by modifying the diffused step profile, 2) a simple processing technique and compatible with existence fabrication technologies of the semiconductor lasers, and 3) enhances the yield-rate of single longitudinal mode operation of the $\lambda / 4$ DFB lasers.

\section{REFERENCES}

[1] H. Soda, Y. Kotaki, H. Sudo, H. Ishikawa, S. Yamakoshi, and H. Imai, "Stability in single longitudinal mode operation in GaInAsP/InP phaseadjusted DFB lasers," IEEE J. Quantum Electron., vol. QE-23, pp. $804-814,1987$.

[2] J. E. A. Whiteaway, G. H. B. Thompson, A. J. Collar, and C. J. Armistead, "The design and assessment of $\lambda / 4$ phase-shifted DFB laser structure," IEEE J. Quantum Electron., vol. 25, pp. 1261-1279, 1989.

[3] E. H. Li, B. L. Weiss, K. S. Chan, and J. Micallef, "The polarization depencent refractive index of an interdiffusion induced AlGaAs/GaAs quantum well," Appl. Phys. Lett., vol. 62, pp. 550-552, 1992.

[4] E. H. Li and K. S. Chan, "Laser gain and current density in a disordered AlGaAs/GaAs quantum well," Electron. Lett., vol. 29, pp. 1233-1224, 1993.

[5] E. H. Li, B. L. Weiss, and S. K. Chan, "Effect of interdiffusion on the subband structure in an AIGaAs/GaAs single quantum well structure," Phys. Rev. B, vol. 46, pp. 15181-15191, 1992.

[6] C. H. Herny, R. A. Logan, and K. A. Bertness, "Spectral dependence of the change in refractive index due to carrier injection in GaAs lasers," J. Appl. Phys., vol. 52, pp. 4457-4461, 1981.

[7] S. R. Andrew, J. H. Marsh, M. C. Holland, and A. H. Kean, "Quantum well laser with integrated passive waveguide fabrication by neutral impurity disordering," IEEE Photon. Technol. Lett., vol. 4, pp. 426-428, 1992.

[8] W. B. Joyce and R. W. Dixon, "Thermal resistance of heterstructure lasers," 'J. Appl. Phys., vol. 46, pp. 855-862, 1975. 